\title{
Multifunctional Molecular Modulation for Efficient and Stable Hybrid Perovskite Solar Cells
}

\author{
Jovana V. Milićsa, Dominik J. Kubicki ${ }^{b}$, Lyndon Emsley ${ }^{b}$, and Michael Grätzela \\ §SCS-DSM Award for best poster presentation in Physical Chemistry
}

\begin{abstract}
Hybrid organic-inorganic perovskites have become one of the leading thin-film semiconductors for optoelectronics. Their broad application will greatly depend on overcoming the key obstacles associated with poor stability and limited scalability. There has been an ongoing effort to diminish some of these limitations by using organic additives. However, considering the lack of understanding of the underlying structure-property relationships, this progress was greatly based on trial and error as molecular-level design remains challenging. Our approach for enhancing the stability of hybrid perovskites without compromising their efficiency is based on judicious molecular design of multifunctional molecular modulators through fine-tuning of noncovalent interactions and exploiting their structural adaptability. The design principles were scrutinized by solid-state NMR spectroscopy to unravel a new path for stable and scalable perovskite solar cells, which we review in this article.
\end{abstract}

Keywords: Hybrid perovskites · Molecular engineering $\cdot$ Molecular modulation $\cdot$ Solar cells $\cdot$ Solid-state NMR

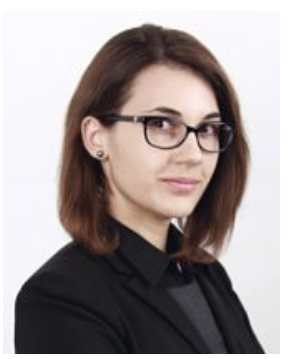

Dr. Jovana $\boldsymbol{V}$. Milić obtained her $\mathrm{PhD}$ in the group of Prof. François Diederich in the Department of Chemistry and Applied Biosciences at ETH Zurich in July 2017. Since October 2017, she works as a scientist with Prof. Michael Graetzel on the development of photovoltaic materials in the Laboratory for Photonics and Interfaces at EPFL, with current focus on dye-sensitized and perovskite solar cells. Her research encompasses (supra)molecular engineering of molecular machines and bioinspired organic materials with the aim of developing functional nanotechnologies for energy conversion. For more information, refer to her LinkedIn profile (linkedin.com/in/ jovanavmilic), ORCID (0000-0002-9965-3460), and Twitter (@ jovana_v_milic).

\section{Introduction}

One of the most promising solutions to the increasing energy demands relies on the conversion of the energy of light into electricity by photovoltaic (PV) technologies. ${ }^{[1,2]}$ With this objective, a new generation of solution-processable thin-film solar cells has recently emerged and has surpassed the market leader, polycrystalline silicon, in less than a decade. ${ }^{[3]}$ These technologies, known as perovskite solar cells (PSCs), are based on hybrid organic-inorganic perovskite semiconductors and feature solar-to-electric power conversion efficiencies (PCE) presently exceeding $23 \% .^{[4]}$ While the progress of PSCs is remarkable, they face a major obstacle to commercial application resulting from the limited stability against environmental factors, such as moisture and oxygen, as well as thermal and electrical stress. ${ }^{[3,5]}$ Moreover, the underlying degradation mechanisms and the corresponding structure-property relationships are not well understood, hampering rational material design that would guide the technological advancement. ${ }^{[3,5]}$ Herein, we review an alternative strategy aiming at overcoming these obstacles based on molecular-level design of multifunctional molecular modulators (MMMs) in conjunction with solid-state NMR spectroscopy to unravel the underlying properties and advance perovskite solar cell research.

\subsection{Hybrid Perovskite Solar Cells}

Hybrid organic-inorganic perovskite semiconductors with the $\mathrm{AMX}_{3}$ (Fig. 1, top) stoichiometry possess a structure composed of a monovalent cation $\mathrm{A}\left(\mathrm{Cs}^{+}\right.$, methylammonium (MA) $\mathrm{CH}_{3} \mathrm{NH}_{3}^{+}$, formamidinium (FA) $\mathrm{CH}\left(\mathrm{NH}_{2}\right)_{2}^{+}$, or guanidinium (GUA) $\left.\mathrm{C}\left(\mathrm{NH}_{2}\right)_{3}^{+}\right)$, a metal $\mathrm{M}\left(\mathrm{Pb}^{2+}, \mathrm{Sn}^{2+}\right)$, and a halide $\mathrm{X}\left(\mathrm{Cl}^{-}\right.$, $\mathrm{Br}^{-}$, or $\left.\mathrm{I}^{-}\right) .{ }^{[3,5-12]}$ They allow for outstanding performances in various optoelectronic devices, including the perovskite solar cells with PCE record exceeding 23\%.[4] However, the instability of perovskite materials towards oxygen, and moisture, as well as electrical and thermal stress, continue to pose substantial challenges. ${ }^{[12-15]}$ While the underlying mechanisms of degradation are not entirely well understood, the reactivity towards water and oxygen are apparent external factors, and so is thermal degradation that appears to result from the internal ion migration. ${ }^{[16-18]}$ There is an ongoing effort to overcome these obstacles based on various strategies, such as compositional engineering, ${ }^{[19,20]}$ as well as employing different additives to enhance the stability. ${ }^{[21-33]}$ However, this approach does not provide clear structure-property relationships, while most of the attempts to enhance the stability come at the expense of the overall performance, posing a requirement for a different strategy.

\footnotetext{
${ }^{*}$ Correspondence: Dr. J. V. Milića

aLaboratory of Photonics and Interfaces, Institut des Sciences et Ingénierie Chimiques, École Polytechnique Fédérale de Lausanne, CH-1015 Lausanne; 'Laboratory of Magnetic Resonance, Institut des Sciences et Ingénierie Chimiques, École Polytechnique Fédérale de Lausanne, $\mathrm{CH}-1015$ Lausanne
} 

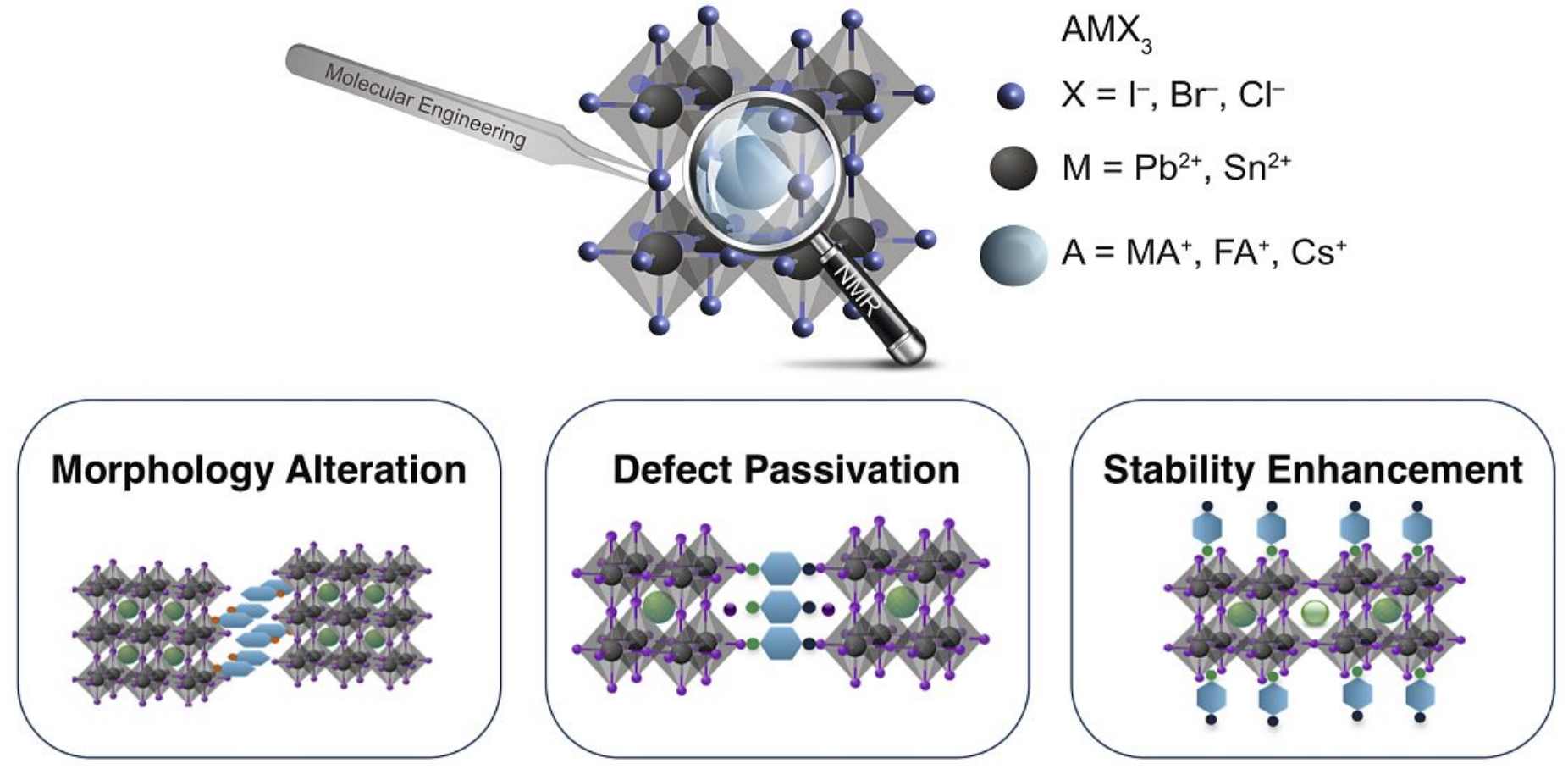

Fig. 1. Top: Schematic of the key tools for molecular modulation illustrated as tweezers and a magnifying glass that represent molecular engineering and solid-state NMR spectroscopy, respectively, highlighting the crystal structure of hybrid perovskites. The structure is based on the $\mathrm{AMX}_{3}$ general formula $\left(\mathrm{A}^{+}=\right.$central cation $\left(\mathrm{Cs}^{+}, \mathrm{MA}^{+}, \mathrm{FA}^{+}\right) ; \mathrm{M}^{2+}=$ divalent metal cation $\left(\mathrm{Pb}^{2+}, \mathrm{Sn}^{2+}\right) ; \mathrm{X}=$ halide $\left.\left(\mathrm{I}^{-}, \mathrm{Br}, \mathrm{Cl}\right)\right)$. Bottom: Schematic overview of the main effects achieved through molecular modulation of hybrid perovskite solar cells, namely morphology alteration (e.g. via crosslinking of perovskite phases), defect passivation (e.g. interaction with uncoordinated metal or halide ions), and stability enhancement (e.g. inert sealing of the perovskite surface).

\subsection{Multifunctional Molecular Modulators}

To overcome the ongoing challenge associated with the stability of hybrid perovskite solar cells without compromising the performance, we have recently introduced the concept of molecular modulation. ${ }^{[34]}$ This refers to designing organic molecules based on the principles of supramolecular chemistry through fine-tuning of noncovalent interactions with the aim of addressing a specific function of the material at the molecular level. Based on the role of a variety of formerly reported organic agents, we could identify three key functional areas that are subject to molecular modulation (Fig. 1, bottom). Firstly, morphology alteration through modulation is desired, as the absorber film morphology dramatically affects the device performance. ${ }^{[35-37]}$ Furthermore, passivation of defects is needed as they might serve as recombination centres, reducing the number of charge carriers and, consequently, the resulting performance. ${ }^{[31,38,39]}$ Finally, stabilization of the perovskite against environmental factors (i.e. via surface encapsulation) or internal ion migration (i.e. through 'blocking layers') is required for further improvement. ${ }^{[16,40]}$ Each of these functions can be translated into a set of complementary strategies addressable via molecular modulation by using noncovalent interactions to alter the morphology, passivate the defects, as well as self-assemble layers for either encapsulation or suppressing detrimental ion migration. Ideally, a molecular modulator should be able to address multiple functions simultaneously, and based on this objective, we have conceptualized multifunctional molecular modulators (MMMs) that meet these requirements, as exemplified by the following case study.

\section{Multifunctional Molecular Modulation: A Case Study}

We have envisaged a multifunctional molecular modulator that would interact with the surface of the hybrid perovskite, while featuring the ability to passivate some of the defects. To that end, we introduced an MMM featuring an ammonium $\left(-\mathrm{NH}_{3}^{+}\right)$function as part of the anilinium core (abbreviated as N; purple in Fig. 2a) that would act as a hydrogen bond-donating site for interaction with the A-cation vacancy on the surface of the hybrid perovskite.
Furthermore, a thiol-tetrazolium unit was introduced into the scaffold (abbreviated as S; grey in Fig. 2a) to enable coordination of the uncoordinated $\mathrm{Pb}^{2+}$ cations that can act as recombination centres that are detrimental to the perovskite solar cell performance. Finally, linking the two units together resulted in the bifunctional 3-(5-mercapto-1H-tetrazol-1-yl)benzenaminium iodide modulator (SN) stabilized in the thione tautomeric form, revealing a supplementary $-\mathrm{NH}$ group for potential interaction with the perovskite surface as part of the hydrophobic heteroaromatic core (Fig. 2a). In order to scrutinize the envisaged mode of action and its effect on the properties of the resulting hybrid perovskite materials, we have also probed two model systems, namely $\mathrm{N}$ and $\mathrm{S}$. Moreover, the study was based on the simplified double cation single halide perovskite composition featuring more thermally stable $\mathrm{FA}^{+}$cations in conjunction with the $\mathrm{Cs}^{+}$cations that facilitate the formation of the $\alpha-\mathrm{FAPbI}_{3}$ perovskite phase. ${ }^{[41-43]}$ The perovskite films were prepared via the vacuum-flash-assisted solution processing (VASP) method.[44] The effect of modulation was investigated by using the precursor solution based on the $(\mathrm{FAI})_{0} \mathrm{Cs}_{01}\left(\mathrm{PbI}_{2}\right)_{105}$ perovskite composition, comprising $\mathrm{FA}_{09} \mathrm{Cs}_{0.1} \mathrm{PbI}_{3}$ with $5 \mathrm{~mol} \%$ excess $\mathrm{PbI}_{2}$, which was modulated with $\mathrm{S}, \mathrm{N}$ and $\mathrm{SN}$ according to the conventional thin-film fabrication procedure. ${ }^{[34]}$ The impact of modulation on perovskite film morphology, structural and optoelectronic properties, as well as photovoltaic performance and stability, was investigated by a combination of techniques involving scanning electronic microscopy (SEM), cathodoluminescence (CL), X-ray photoelectron (XPS), Fourier transformed infrared (FTIR) and photoluminescence (PL) spectroscopy, as well as solid-state NMR spectroscopy.

\subsection{From Morphology Modulation to Defect Passivation}

The effect of molecular modulation on perovskite film morphology was probed by SEM (Fig. 2b). The analysis of the topview SEM images clearly indicates changes of morphology upon modulation, which was reflected in the gradual increase of the grain sizes from about $300 \mathrm{~nm}$ in the control films up to $1 \mu \mathrm{m}$ for the films containing the SN modulator. This was accompanied 
a)

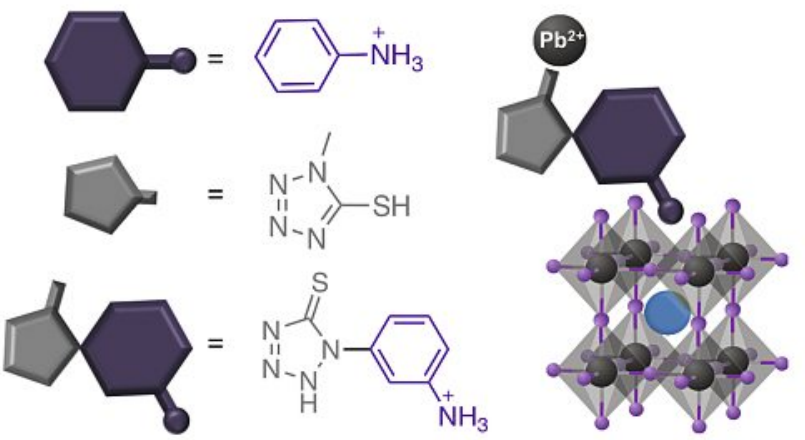

b)

c)
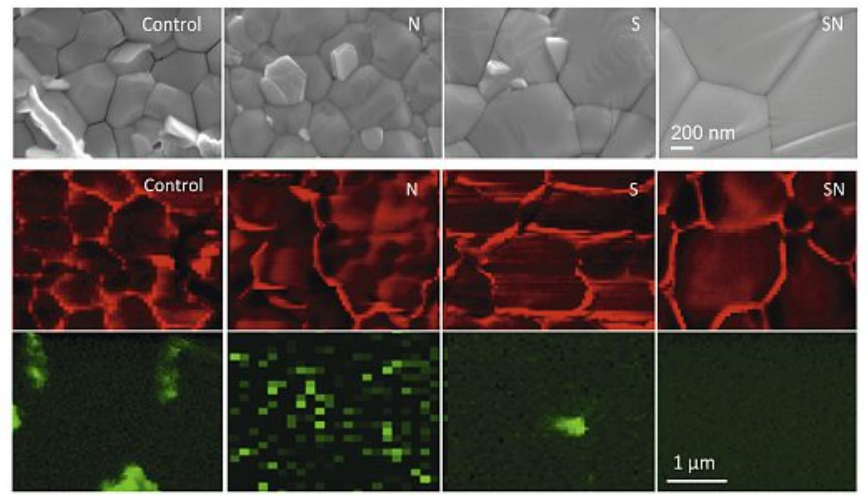

Fig. 2. (a) Schematic representation of the structure of molecular modulators $\mathrm{N}$ (purple), S (grey), and SN (purple-grey) illustrating the interaction of $\mathrm{SN}$ with $\mathrm{Pb}^{2+}$ ions (grey sphere) and perovskite crystal structure (FAPbl ${ }_{3}$, where $\mathrm{FA}=$ blue sphere, $\mathrm{Pb}^{2+}$ and $\mathrm{I}^{-}=$grey and purple spheres, respectively). (b) Top-view SEM images of the pristine (control) and modulated (N, S, and $\mathrm{SN}$ ) perovskite films deposited on mesoporous- $\mathrm{TiO}_{2} /$ compact-TiO /FTO substrates. (c) Cathodoluminescence mapping of the pristine (control) and modulated (N, S, and SN) perovskite films deposited on indium tin oxide (ITO) glass. The spatial distribution of light emission in the 700-800 $\mathrm{nm}$ (red) and 530-590 nm (green) range. Figure adapted with permission of Nature. ${ }^{[34]}$

by the gradual increase in the homogeneity of the film surface, as the number of bright nanoplates observed within the grain boundaries gradually decreased in presence of modulators $\mathrm{S}$ and $\mathrm{N}$, and completely disappeared upon $\mathrm{SN}$ treatment. The nature of these inhomogeneities was further evaluated via CL mapping of the film deposited on a thin indium-tin-oxide (ITO) coated glass substrate (Fig. 2c). The mapping was performed in two spectral regions, from 530 to $590 \mathrm{~nm}$ (in green) and from 700 to $800 \mathrm{~nm}$ (in red). While the latter emission typically corresponds to the hybrid perovskite and is pronounced within the grain boundaries, the $530-590 \mathrm{~nm}$ emission is characteristic of $\mathrm{PbI}_{2}$ and corresponds to the white nanoplates observed in the SEM images. In accordance with the design principles, the quantity of such $\mathrm{PbI}_{2}$ crystallites is reduced in the presence of sulphur-containing species, which is associated with their coordination. This mode of action was further assessed by XRD, XPS, and FTIR analysis (Fig. 3). ${ }^{[34]}$

XRD measurements of the perovskite films revealed a trigonal perovskite phase with the dominant (111) reflexion at $2 \theta$ of $14.15^{\circ}$ as well as gradual disappearance of the signal at $12.5^{\circ}$ that arises from the (001) lattice reflection of hexagonal $\mathrm{PbI}_{2}$ (Fig. 3a). This, however, does not lead to the appearance of any additional phases, despite the use of excess $\mathrm{PbI}_{2}$ in the preparation, which suggests that the reduced $\mathrm{PbI}_{2}$ emission in the $\mathrm{CL}$ measurements is the result of $\mathrm{PbI}_{2}$ possibly becoming amorphous and thus undetectable upon $\mathrm{SN}$-modulation. This could also occur as a result of intercalation of the modulator into the $\mathrm{PbI}_{2}$ lattice leading to the formation of a layered two-dimensional structure. While the intensity of the XRD signal corresponding to $\mathrm{PbI}$, reduced for $\mathrm{N}$ - and $\mathrm{S}$-modulated perovskite structures, the peak entirely disappeared for the $\mathrm{SN}$-modulated
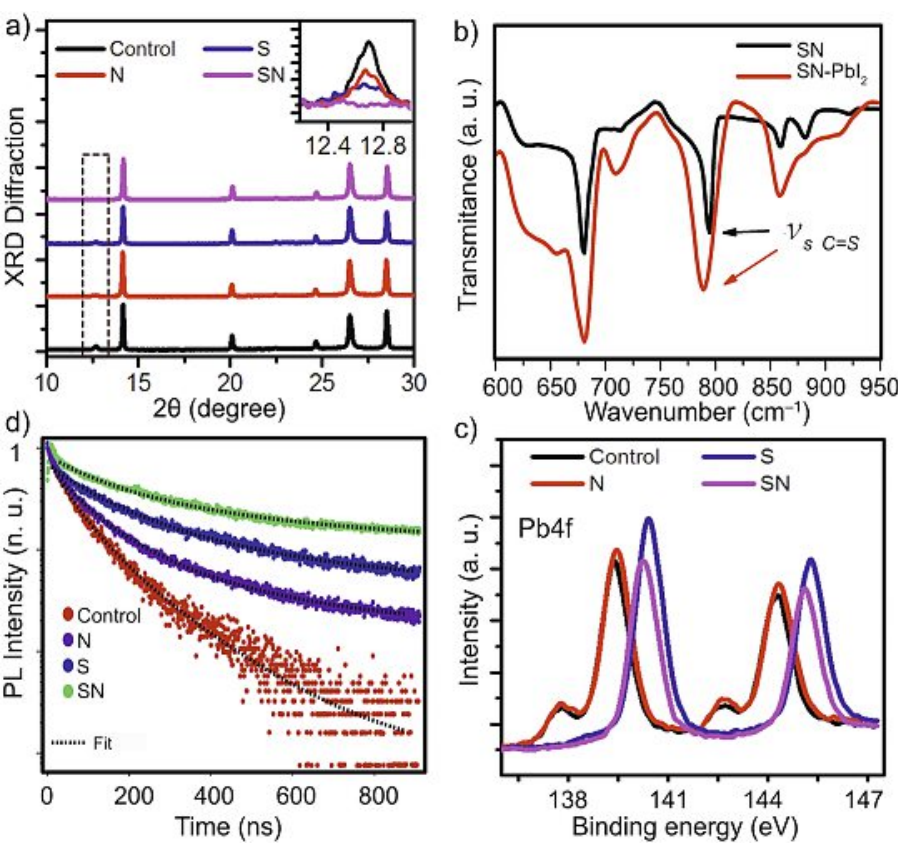

Fig. 3. Material characterization in the absence (control) and presence of molecular modulators (N, S, and SN). (a) XRD patterns with the peak at $12.5^{\circ}$ that arises from the (001) lattice planes of $\mathrm{Pbl}_{2}$ shown in the inset. (b) FTIR and (c) XPS spectra. (d) TRPL decays (the dashed lines show the fits of the experimental data). Figure adapted with permission of Nature. ${ }^{[34]}$

film, in line with the effective $\mathrm{PbI}_{2}$ coordination. The involvement of the thione group in the coordination was evidenced by the shift of the $\mathrm{C}=\mathrm{S}$ vibration from $794 \mathrm{~cm}^{-1}$ in $\mathrm{SN}$ to $788 \mathrm{~cm}^{-1}$ upon interaction with $\mathrm{PbI}_{2}$ (Fig. 3b), indicating the formation of an $\mathrm{SN}-\mathrm{PbI}_{2}$ complex that affects the $\mathrm{C}=\mathrm{S}$ bond strength. This was further in accordance with the XPS analysis of the films on mesoporous- $\mathrm{TiO}_{2} /$ compact- $\mathrm{TiO}_{2} / \mathrm{FTO}$ substrates (Fig. 3c). The XPS spectra of the control sample and the modulated perovskite films feature symmetric peaks with comparable spin-orbit splitting between the $\mathrm{Pb} 4 f 7 / 2$ and $\mathrm{Pb} 4 f 5 / 2$ levels in all cases. The $\mathrm{Pb} 4 f 7 / 2$ level signals shifted by approximately $0.9 \mathrm{eV}$ to higher binding energy upon modulation by using either S or SN, which can be ascribed to the bond formation with the sulphur moieties. Since XPS probes mainly the surface species, the $\mathrm{Pb}$ signal at around $139.5 \mathrm{eV}$ was ascribed to coordinatively unsaturated $\mathrm{Pb}^{2+}$ surface ions. In addition, the spectra of the control and $\mathrm{N}$-modulated samples also reveal smaller peaks at $137.8 \mathrm{eV}$ and $142.6 \mathrm{eV}$ that can be attributed to other $\mathrm{Pb}$ (II) species, such as $\mathrm{PbO}$, which can form as an oxidative decomposition product at the interface. ${ }^{[45]}$ This might result from the exposure to traces of oxygen and water in the environment.

$\mathrm{The} \mathrm{Pb}(\mathrm{II})$ species revealed by XPS can act as defects, serving as non-radiative recombination centres that reduce the photovoltaic performance, hence their elimination is critical to realizing perovskite films of high electronic quality. The suppression of non-radiative recombination would result in the increase of the PL lifetimes, which can be probed by time-resolved photoluminescence (TRPL) spectroscopy. We examined the evolution of the photoluminescence (PL) emission at $790 \mathrm{~nm}$ using N-, S- and SNmodulated perovskite films (Fig. 3d). The decay of the PL intensity corresponds to the number of photons emitted by the perovskite per unit time and fitting the decays provides the lifetimes for the first order non-radiative PL decay process. The longest lifetimes of $2.770 \mu$ s were apparent for the $\mathrm{SN}$-modulated perovskite films, which is exceptionally long for metal halide perovskites. This clearly indicates passivation of defects and, consequently, the improvement of electronic quality of the perovskite materials. ${ }^{[34]}$ Since modulators $\mathrm{S}$ and $\mathrm{SN}$ reduce the density of uncoordinated 
$\mathrm{Pb}^{2+}$ sites, they are more effective in passivating the surface defects of the perovskite films.

While the outlined study of the thin films provides important indicators of the mode of action of the corresponding modulators, the assessment of the general structure-property relationships requires analysis of atomic-level interactions, which can be uniquely performed by means of solid-state NMR spectroscopy.

\subsection{Structural Properties upon Molecular Modulation}

We carried out solid-state NMR experiments on several nuclei, including ${ }^{13} \mathrm{C},{ }^{14} \mathrm{~N}$, and ${ }^{15} \mathrm{~N}$, and compared the corresponding magic angle spinning (MAS) NMR spectra of neat $\mathrm{PbI}_{2}$ and bulk mechanochemical $\alpha-\mathrm{FAPbI}_{3}$ as a model perovskite compound to those of the bulk mechanochemical mixtures of either $\alpha-\mathrm{FAPbI}_{3}$ or $\mathrm{PbI}_{2}$ with $4 \mathrm{~mol} \% \mathrm{SN} .{ }^{[46-49]}$ As a result, we obtained three key structural insights.

First, the presence of interactions between the modulator and either the perovskite or $\mathrm{PbI}_{2}$ can be evidenced by the new ${ }^{13} \mathrm{C}$ NMR resonances in the corresponding mechanochemical mixtures. Indeed, a clear difference between the ${ }^{13} \mathrm{C}$ NMR spectra of the $\mathrm{SN}$-modulated $\alpha-\mathrm{FAPbI}_{3}$ perovskite (Fig. 4a) and neat $\mathrm{SN}$ (Fig. 4b) can be observed, revealing a set of additional peaks that are compatible with SN in a new environment. Specifically, the aromatic peaks are shifted and broadened in the ranges between $110-120$ as well as $135-140 \mathrm{ppm}$, and similarly, the $\mathrm{C}=\mathrm{S}$ peak is shifted and broadened to the range between $165-170 \mathrm{ppm}$. These carbon environments can be ascribed to the SN interacting with the surface of the $\alpha-\mathrm{FAPbI}_{3}$ perovskite phase. Similarly, the interaction of $\mathrm{SN}$ with $\mathrm{PbI}_{2}$ was evidenced.

Furthermore, the chemical shifts can provide information on whether the interaction occurs at the surface of the perovskite or by incorporation into the corresponding A cation sites. For this purpose, we acquired ${ }^{13} \mathrm{C}$ and ${ }^{15} \mathrm{~N}$ NMR spectra of the pristine and modulated $\alpha-\mathrm{FAPbI}_{3}$ material. The main perovskite phase in both cases yields similar ${ }^{13} \mathrm{C}$ and ${ }^{15} \mathrm{~N}$ resonances (Fig. $4 \mathrm{c}, \mathrm{d}$ and Fig. 4e,f, respectively). This strongly indicates that SN does not incorporate into the perovskite lattice. This is not surprising as the largest cation which has been shown to date to incorporate into the $\alpha-\mathrm{FAPbI}_{3}$ lattice is guanidinium, ${ }^{[48]}$ which is substantially smaller than $\mathrm{SN}$, hence its incorporation into the bulk of the $3 \mathrm{D}$ perovskite structure is unlikely. However, a small shift of about $+0.2 \mathrm{ppm}$ in both ${ }^{13} \mathrm{C}$ and ${ }^{15} \mathrm{~N}$ NMR spectra upon $\mathrm{SN}$ modulation indicates the presence of structural differences between the two materials.

Finally, these structural features of the modulated 3D perovskite phase can be further assessed by ${ }^{14} \mathrm{~N}$ NMR. The ${ }^{14} \mathrm{~N}$ MAS NMR spectra of $\alpha-\mathrm{FAPbI}_{3}$ (Fig. 4g,i) and $\alpha-\mathrm{FAPbI}_{3}$ with $4 \mathrm{~mol} \% \mathrm{SN}$ (Fig. $4 \mathrm{~h}, \mathrm{j})$ feature a spinning sideband (SSB) pattern that is narrower in the SN-modulated material. It has been shown that the breadth of the residual ${ }^{14} \mathrm{~N}$ SSB manifolds is affected by the reorientation of FA in the fast motion regime that is related to the symmetry of cation reorientation inside the cuboctahedral perovskite cavity, such as that narrower ${ }^{14} \mathrm{~N} \mathrm{SSB}$ manifolds indicate higher symmetry that is closer to cubic. ${ }^{[46]}$ This suggests that the crystallographic symmetry of the $\alpha-\mathrm{FAPbI}_{3}$ phase is affected by modulation. However, the isotropic peak has the same shift in both samples, suggesting that the structural differences are subtle, which supports the conclusion that the change in the crystallographic symmetry of the $\alpha-\mathrm{FAPbI}_{3}$ phase is not caused by cation incorporation but a surface interaction. Furthermore, this suggests that $\mathrm{SN}$ acts as a structure-directing agent that promotes growth of a modified perovskite structure, as compared to that of untreated $\alpha-\mathrm{FAPbI}_{3}$. In a nutshell, the solid-state NMR results show that the modulation of the perovskite with SN as a molecular modulator tunes the morphology of the perovskite layer through a structure-directing role by binding to the perovskite surface, while simultaneously passivating the defects, which is in accordance with its multifunctionality that benefits both the device performance and stability.
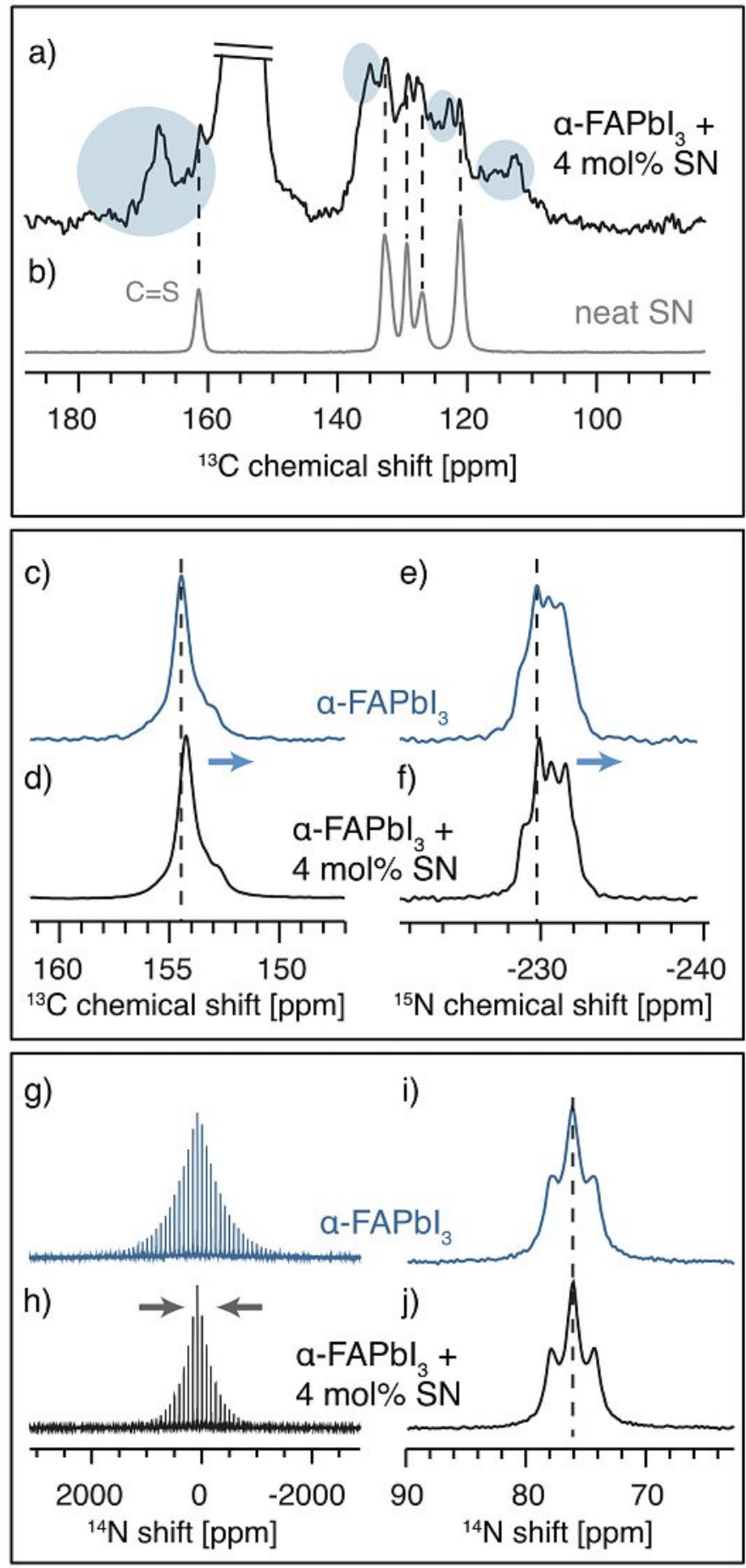

Fig. 4. (a-b) ${ }^{13} \mathrm{C}$ CP solid-state MAS NMR spectra at $11.7 \mathrm{~T}, 105 \mathrm{~K}$ and $10 \mathrm{kHz}$ MAS of a) bulk mechanochemical $\alpha$-FAPbl ${ }_{3}$ with $4 \mathrm{~mol} \% \mathrm{SN}$ and b) neat $\mathrm{SN}$ powder (in the SN spectral region). Blue circles highlight the new environments that are associated with the interaction of $\mathrm{SN}$ with the perovskite. (c-f) ${ }^{13} \mathrm{C} \mathrm{CP}$ and ${ }^{15} \mathrm{~N}$ CP solid-state MAS NMR spectra at $11.7 \mathrm{~T}, 105 \mathrm{~K}$ and $10 \mathrm{kHz}$ MAS of bulk mechanochemical c,e) $\alpha-\mathrm{FAPbl}_{3}$ and d,f) $\alpha-\mathrm{FAPbl}_{3}$ with $4 \mathrm{~mol} \% \mathrm{SN}$ (in the FA spectral region). Blue arrows indicate a minor chemical shift $(0.2 \mathrm{ppm}) .(\mathrm{g}-\mathrm{j}){ }^{14} \mathrm{~N}$ solid-state MAS NMR spectra at $11.7 \mathrm{~T}, 298 \mathrm{~K}$ and g,h) $3 \mathrm{kHz}$ and i,j) $20 \mathrm{kHz}$ MAS of bulk mechanochemical g) $\alpha$-FAPbl 3 and h) $\alpha$-FAPbl ${ }_{3}$ doped with 4 mol\% SN; whereas $\mathrm{i}, \mathrm{j}$ ) shows the corresponding close-up views of the center band. The triplet results from the ${ }^{1} \mathrm{H}-{ }^{14} \mathrm{~N} \mathrm{~J}$-coupling. Grey arrows highlight the apparent narrowing of the SSB manifold. Adapted from ref. [34] with permission from Nature.

\subsection{Photovoltaic Performance and Stability}

The beneficial properties of molecular modulation were assessed in photovoltaic devices by fabricating the corresponding solar cells of the conventional $\mathrm{Au} / 2,2^{\prime}, 7,7^{\prime}$-tetrakis $(N, N$-di- 
pmethoxyphenylamine)-9,9-spirobifluorene (spiro-OMeTAD)/ perovskite/mesoporous- $\mathrm{TiO}_{2} /$ compact- $\mathrm{TiO}_{2} /$ fluorine-doped tin oxide (FTO) architecture with an active area of $1.44 \mathrm{~cm}^{2}$ and aperture of $1 \mathrm{~cm}^{2}$. Moreover, the reproducibility of the approach was assessed by preparing 40 cells, which were measured under comparable conditions of standard AM 1.5G illumination at light intensity of $100 \mathrm{~mW} \mathrm{~cm}{ }^{-2}$ (Fig. 5a). As a result, the effect of modulation was evident, showing an increase of all the photovoltaic parameters as compared to the control sample, including short circuit current density $\left(J_{\mathrm{SC}}\right)$, open-circuit voltage $\left(V_{\mathrm{OC}}\right)$, fill factor (FF), and PCE, accompanied by lower hysteresis (Fig. 5a). While the $J_{\mathrm{SC}}$ improvements can be related to the better electronic quality of the films and more effective charge collec- tion in presence of the modulators, the $V_{\mathrm{OC}}$ enhancement results from the suppression of charge carrier recombination as a result of the defect passivation. The effects were most significant in case of SN-modulated samples showing up to $20.9 \%$ PCE, with $J_{\mathrm{SC}}$ of $24 \mathrm{~mA} \mathrm{~cm}^{-2}, V_{\mathrm{OC}} 1.15 \mathrm{~V}$, and FF of 0.75 (Fig. 5b). The $J_{\mathrm{SC}}$ values were in agreement with the integrated current density derived from the incident photon-to-electron conversion efficiency (IPCE) spectrum, which excludes spectral mismatch between the simulator and the AM 1.5G solar source (Fig. 5c). This exceptional performance reaches beyond the state-of-theart values for double cation single halide perovskite solar cells even for active areas that are almost an order of magnitude larger than the size of a conventional laboratory-scale solar cell $(0.16$ a)
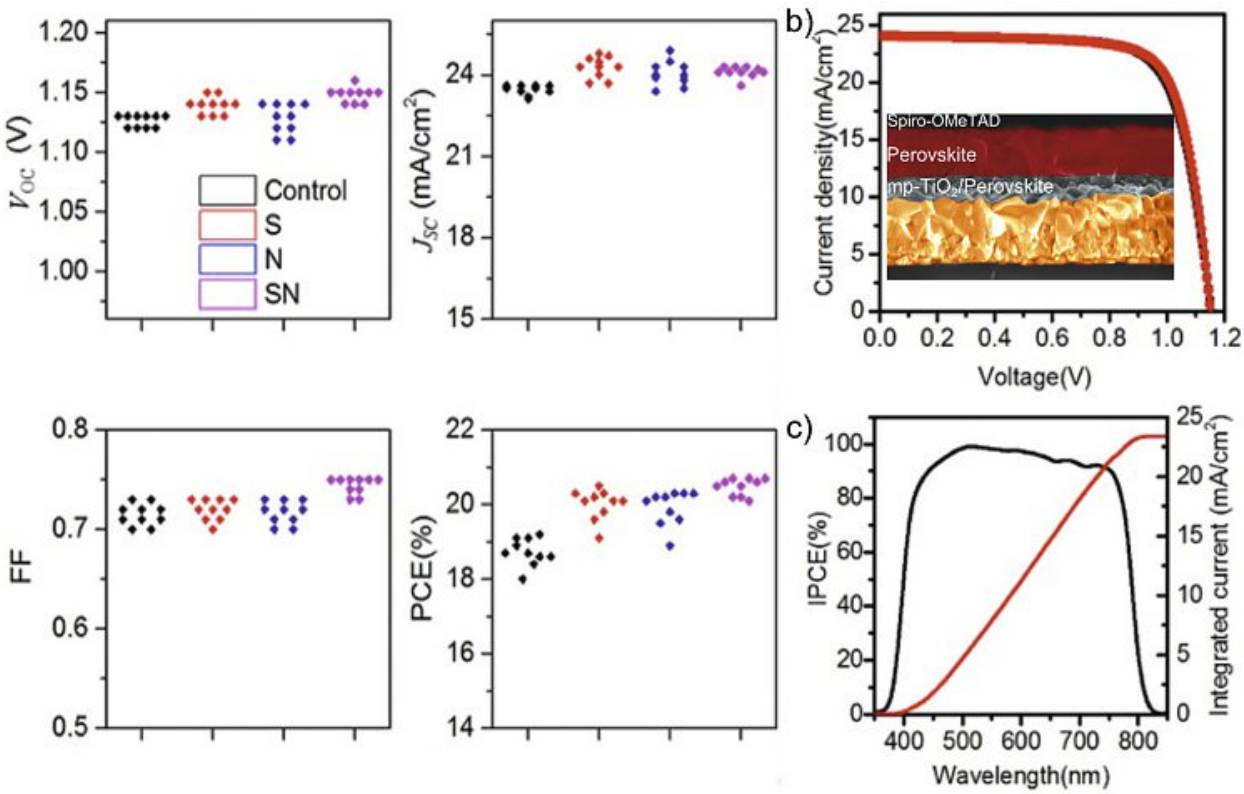
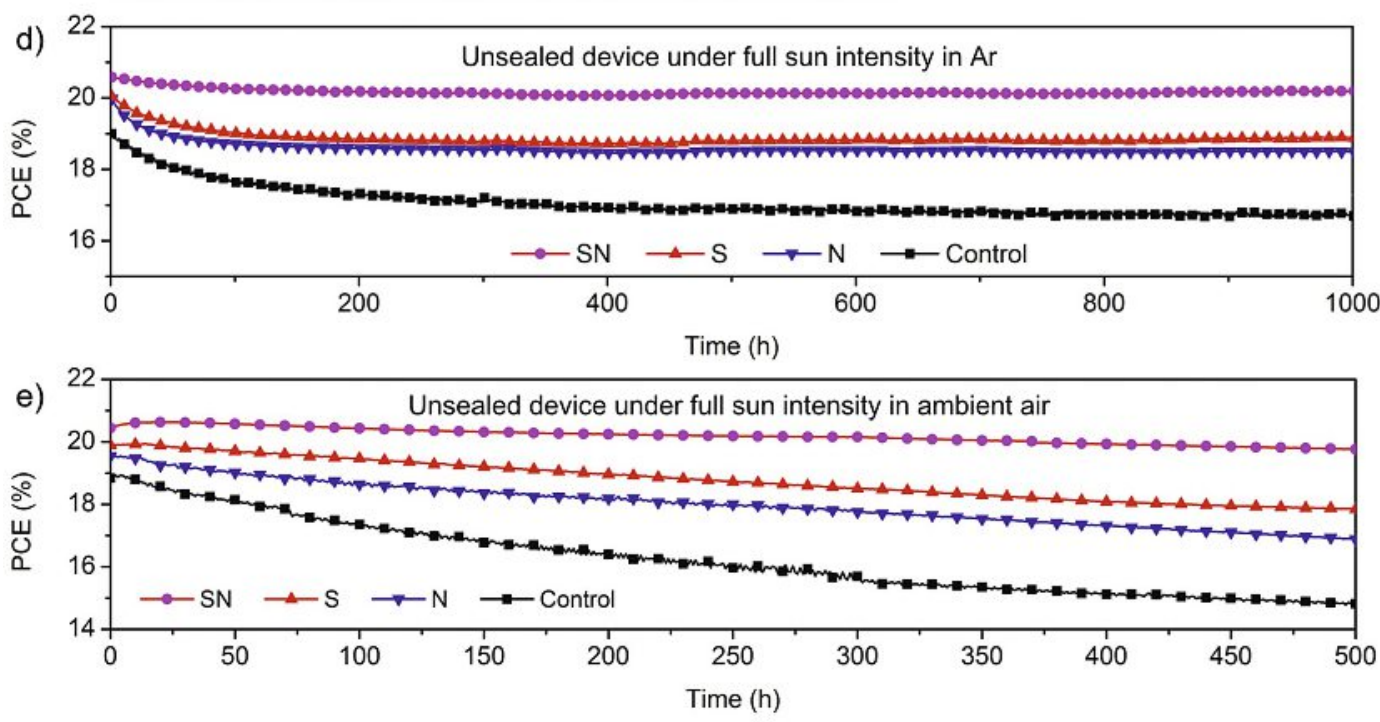

f)
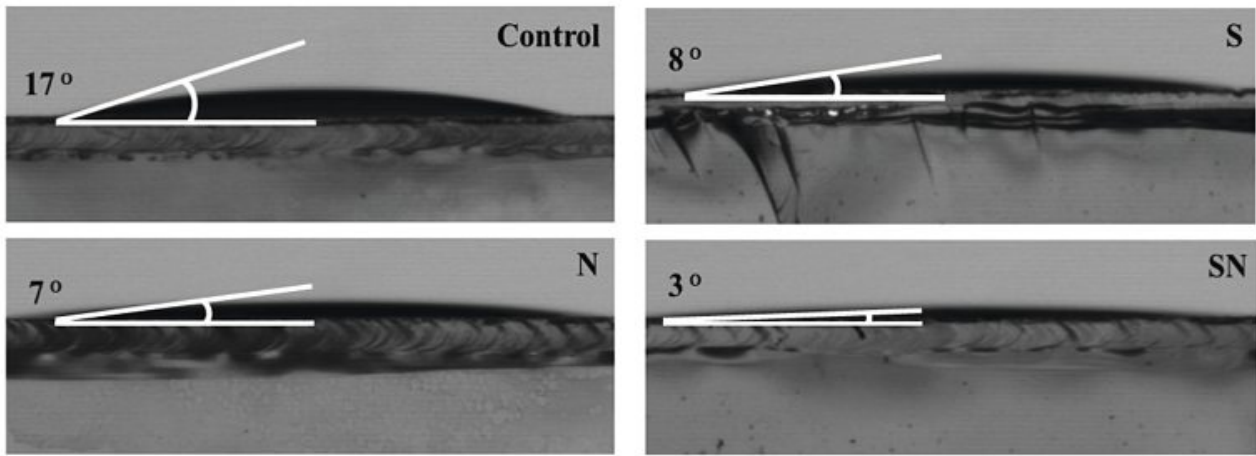

Fig. 5. (a) Photovoltaic metrics of pristine (control) and modulated (S, N, SN) PSCs with aperture areas of $1 \mathrm{~cm}^{2}$. (b) $J-V$ curves of the champion SN-modulated device recorded in reverse (from $V_{\mathrm{OC}}$ to $J_{\mathrm{SC}}$ ) and forward (from $J_{\mathrm{SC}}$ to $V_{\mathrm{OC}}$ ) scanning directions at a scanning rate of $50 \mathrm{mV} \mathrm{s}^{-1}$ under AM 1.5G irradiation. Cross-sectional SEM image of the control device is presented in the inset with the corresponding layers. (c) The IPCE spectrum (black) of the champion SN-modulated device with the projected photocurrent (red) derived from integrating the IPCE over the standard AM $1.5 \mathrm{G}$ spectral range. (d-e) Evolution of the PCE over time measured by MPP tracking of unsealed PSCs with aperture area of $0.16 \mathrm{~cm}^{2}$ upon continuous illumination at temperatures between $55-60{ }^{\circ} \mathrm{C}$ under d) argon and e) ambient air at 20\% humidity. (f) Contact angle measurements of pristine (control) and modulated perovskite films with the droplet of the HTM solution. Adapted from ref. [34] with permission from Nature. 
$\mathrm{cm}^{2}$ ). Such scalability is permitted by the employment of antisolvent-free VASP preparation method in conjunction with the effective modulation of the structure and morphology of perovskite films.

Remarkably, the excellent performance was accompanied by a long-term operational stability probed by maximum power point (MPP) tracking upon continuous full sun illumination under elevated degradation conditions at $55-60{ }^{\circ} \mathrm{C}$ in either inert argon atmosphere (Fig. 5d) or humid ambient air (Fig. 5e). While all the modulators enhanced the operational stability under both conditions, SN enabled maintaining $98.1 \%$ of initial PCE for more than $1000 \mathrm{~h}$ in argon atmosphere and $96.6 \%$ for more than $500 \mathrm{~h}$ in ambient air. This outstanding stability upon modulation can be associated with the suppression of morphological changes that can occur upon aging through interactions with the perovskite surface, as well as passivation of surface defects. Furthermore, hydrophobicity is an important criterion for enhancing the stability in ambient air, which is probed by analysing the contact angle of the hydrophobic chlorobenzene-based hole transporting material (HTM) solution droplet on the modulated films (Fig. 5f). The analysis shows increased hydrophobicity, indicating improved compatibility of the perovskite layer with the HTM. This in turn enables the formation of a compact layer that is instrumental for an efficient charge extraction and essential for the performance. Moreover, increased hydrophobicity results in higher tolerance to critical environmental factors, oxygen and moisture, which further contributes to the durability of the devices.

\section{The Generality of Multifunctional Molecular Modulation in Hybrid Perovskite Solar Cells}

The case of multifunctional molecular modulation illustrated by the role of $\mathrm{SN}$ in hybrid perovskite solar cells implies unique characteristics. This is associated with the presence of multiple functional groups with complementary roles as part of a hydrophobic scaffold that results in a simultaneous improvement of performance and stability through morphology alteration, defect passivation, and enhanced tolerance to environmental factors. The beneficial aspects of both structural and operational multifunctionality are by no means limited to the SN case or the single perovskite composition. On the contrary, this prototype has stimulated a series of other successful developments that show the generality of the approach.

For instance, we have employed an even more hydrophobic carbocyclic scaffold in 1-adamantylammonium halide modulators (ADAHX; Fig. 6a) that produced one of the most general approaches to improving the performance and stability of a variety of different perovskite compositions yielding efficiencies of almost $22 \%$ with remarkable photovoltages up to $1.245 \mathrm{~V} \cdot{ }^{[50]}$ Moreover, the modulation of the perovskite surface was shown to be accompanied by comparable signatures of the interaction of the modulator with the hybrid perovskite revealed by solid-state NMR spectra (Fig. 6b-6g), with particular emphasis on the comparable structure-directing role (Fig. 6f-6g), promoting the crystal structure with higher electronic quality.

Ultimately, the approach of fine-tuning noncovalent interactions through molecular-level engineering probed by solid-state NMR spectroscopy enabled the development of more elaborate hybrid perovskite structures with further enhanced stability. This was achieved by applying the molecular-level design principles to layered two-dimensional perovskites, which feature an organic spacer layer incorporated into the perovskite crystal lattice that is formed by alternating units of the spacer and the hybrid perovskite component (Fig. 6h). Such layered perovskites have already been shown to feature superior stability ${ }^{[51]}$ and their full implementation into highly efficient solar cells based on hybrid perovskite conjugates is underway.

\section{Summary and Outlook}

Hybrid organic-inorganic perovskite solar cells have reached unprecedented success in photovoltaics, while their stability and molecular-level engineering remain challenging. We have demonstrated the effectiveness of the concept of multifunctional molecular modulation in addressing these challenges based on molecular-level design guided by the principles of supramolecular chemistry and elucidated by unravelling atomic-level interactions via solid-state NMR spectroscopy. These studies open a new path for the realization of highly efficient and stable perovskite solar cells based on molecular design in the future.

\section{Acknowledgements}

J.V.M and M.G. are grateful to the Swiss National Science Foundation for financial support of the joint project IZLRZ2_164061 under the Scientific \& Technological Cooperation Program SwitzerlandRussia and the European Union's Horizon 2020 research and innovation program (No. 687008, GOTSolar) and ERC Proof of concept grant (No. 713681, PRINTSolar). D.J.K. and L.E acknowledge support from Swiss National Science Foundation Grant No. 200021_160112. The authors are further grateful to all the co-workers that were involved in the work reviewed in this article.

Received: January 26, 2019

[1] S. Sorrell, Renew. Sust. Energy Rev. 2015, 47, 74.

[2] M. Hosenuzzaman, N. A. Rahim, J. Selvaraj, M. Hasanuzzaman, A. B. M. A. Malek, A. Nahar, Renew. Sust. Energy Rev. 2015, 41, 284.

[3] Y. Rong, Y. Hu, A. Mei, H. Tan, M. I. Saidaminov, S. I. Seok, M. D. McGehee, E. H. Sargent, H. Han, Science 2018, 361, eaat8235.

[4] 'NREL Best Research Cell Efficiency Chart', www.nrel.gov-pv-assets-images-efficiency-chart.png. (accessed in December 2018)

[5] C. H. Ng, H. N. Lim, S. Hayase, Z. Zainal, N. M. Huang, Renew. Sust. Energy Rev. 2018, 90, 248.

[6] J.-P. Correa-Baena, M. Saliba, T. Buonassisi, M. Grätzel, A. Abate, W. Tress, A. Hagfeldt, Science 2017, 358, 739.

[7] N. Pellet, P. Gao, G. Gregori, T.-Y. Yang, M. K. Nazeeruddin, J. Maier, M. Grätzel, Angew. Chem. Int. Ed. 2014, 53, 3151.

[8] M. Grätzel, Nature Mater. 2014, 13, 838.

[9] J.-P. Correa-Baena, A. Abate, M. Saliba, W. Tress, T. J. Jacobsson, M. Grätzel, A. Hagfeldt, Energy Environ. Sci. 2017, 10, 710.

[10] M. Saliba, J.-P. Correa-Baena, M. Graetzel, A. Hagfeldt, A. Abate, Angew. Chem. Int. Ed. 2018, 57, 2554.

[11] M. Grätzel, Acc. Chem. Res. 2017, 50, 487

[12] M. Saliba, T. Matsui, K. Domanski, J-Y. Seo, A. Ummadisingu, S. M Zakeeruddin, J-P. Correa-Baena, W. R. Tress, A. Abate, A. Hagfeldt, M. Grätzel, Science 2016, 354, 206.

[13] Y. H. Lee, J. Luo, R. Humphry-Baker, P. Gao, M. Grätzel, M. K. Nazeeruddin, Adv. Funct. Mater. 2015, 25, 3925.

[14] N. H. Tiep, Z. Ku, H. J. Fan, Adv. Energy Mater. 2016, 6, 1501420.

[15] T. A. Berhe, W.-N. Su, C.-H. Chen, C.-J. Pan, J.-H. Cheng, H.-M. Chen, M.C. Tsai, L.-Y. Chen, A. A. Dubale, B.-J. Hwang, Energy Environ. Sci. 2016, 9, 323 .

[16] K. Domanski, B. Roose, T. Matsui, M. Saliba, S.-H. Turren-Cruz, J.-P. Correa-Baena, C. R. Carmona, G. Richardson, J. M. Foster, F. De Angelis, J. M. Ball, A. Petrozza, N. Mine, M. K. Nazeeruddin, W. Tress, M. Grätzel, U. Steiner, A. Hagfeldt, A. Abate, Energy Environ. Sci. 2017, 10, 604.

[17] K. Domanski, J.-P. Correa-Baena, N. Mine, M. K. Nazeeruddin, A. Abate, M. Saliba, W. Tress, A. Hagfeldt, M. Grätzel, ACS Nano 2016, 10, 6306.

[18] K. Domanski, E. A. Alharbi, A. Hagfeldt, M. Grätzel, W. Tress, Nat. Energy 2018, 3, 61 .

[19] N. J. Jeon, J. H. Noh, W. S. Yang, Y. C. Kim, S. Ryu, J. Seo, S. I. Seok, Nature 2015, 517, 476

[20] W. S. Yang, B.-W. Park, E. H. Jung, N. J. Jeon, Y. C. Kim, D. U. Lee, S. S Shin, J. Seo, E. K. Kim, J. H. Noh, S. I. Seok, Science 2017, 356, 1376.

[21] H. Zhang, M. K. Nazeeruddin, W. C. H. Choy, Adv. Mater. 2019, $2,1805702$.

[22] B.-C. Kang, S. Lee, D.-W. Kang, T.-J. Ha, IEEE El. Dev. Lett. 2018, 39, 843

[23] J. V. Milić, N. Arora, M. I. Dar, S. M. Zakeeruddin, M. Grätzel, Adv. Mater. Interfaces 2018, 356, 1800416

[24] J. Cao, J. Yin, S. Yuan, Y. Zhao, J. Li, N. Zheng, Nanoscale 2015, 7, 9443.

[25] M. M. Tavakoli, D. Bi, L. Pan, A. Hagfeldt, S. M. Zakeeruddin, M. Grätzel, Adv. Energy Mater. 2018, 8, 1800275.

[26] D. Bi, P. Gao, R. Scopelliti, E. Oveisi, J. Luo, M. Grätzel, A. Hagfeldt, M. K. Nazeeruddin, Adv. Mater. 2016, 28, 2910.

[27] D. Bi, C. Yi, J. Luo, J.-D. Décoppet, F. Zhang, S. M. Zakeeruddin, X. Li, A. Hagfeldt, M. Grätzel, Nat. Energy 2016, 1, 16142. 

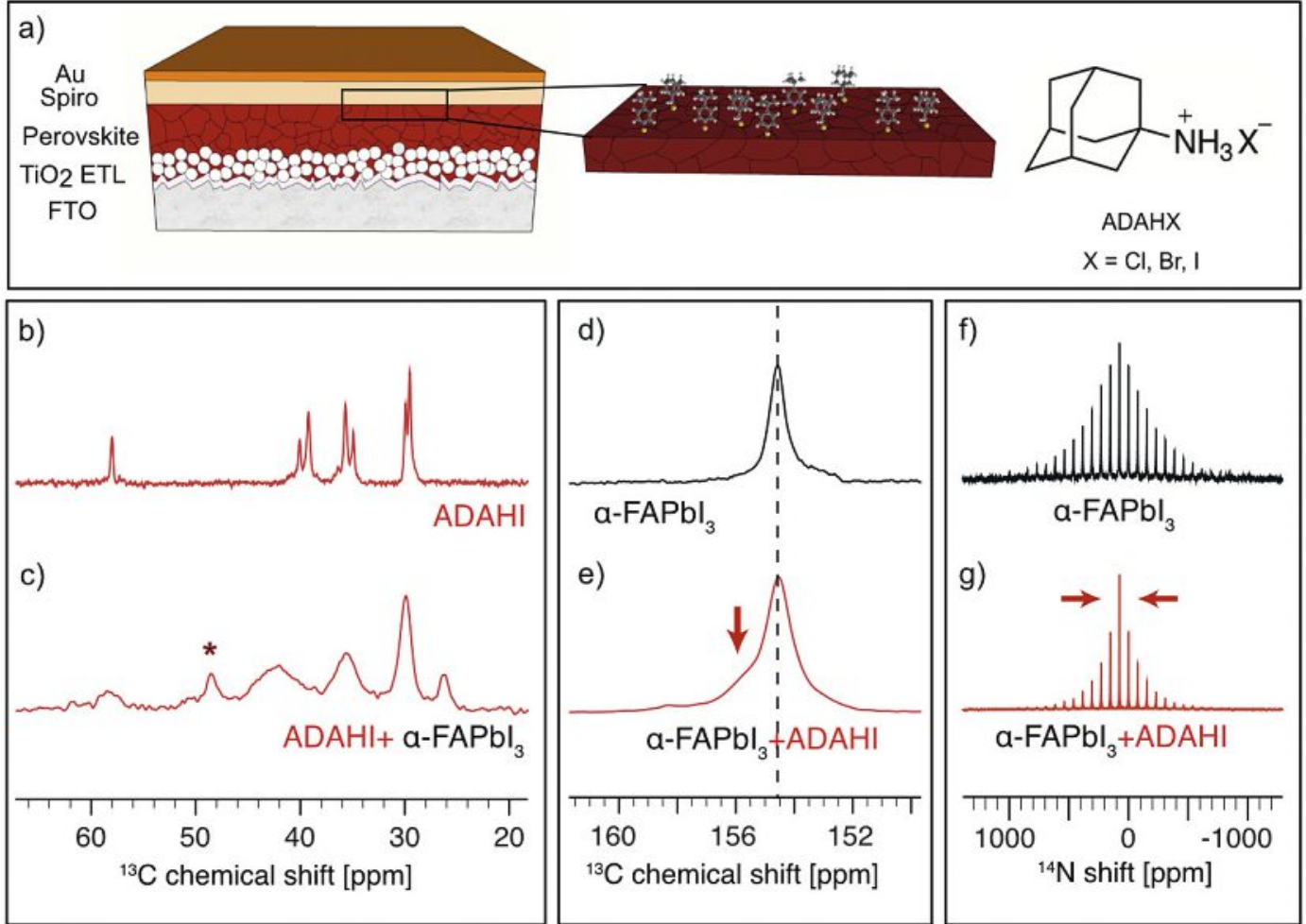

h)

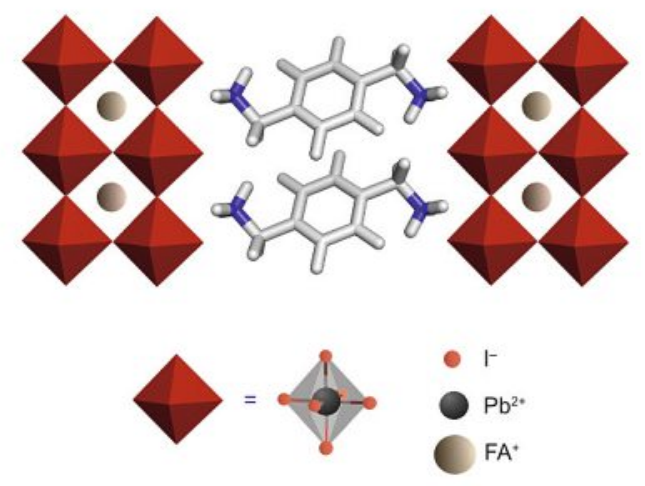

Fig. 6. (a) Schematic representation of the perovskite solar cell with the corresponding modification. Left: Schematic of device architecture indicating the presence of ADAHI on the surface of the perovskite film. Right: Chemical structure of ADAHX. $(\mathrm{b}-\mathrm{g}){ }^{13} \mathrm{C} \mathrm{CP}$ solid-state MAS NMR spectra at 21.1 T, $100 \mathrm{~K}$ and $12 \mathrm{kHz}$ MAS of (b) neat ADAHI powder and (c) $\alpha$-FAPbl ${ }_{3}$ treated with ADAHI (in the ADAHI spectral region), as well as (d) neat $\alpha-\mathrm{FAPbl}_{3}$ and (e) $\alpha$-FAPbl treated with ADAHI (in the FA spectral region). The red arrow indicates a new FA environment present upon modulation while the asterisk indicates a spinning sideband of the FA signal.

${ }^{14} \mathrm{~N}$ solid-state MAS NMR spectra at $21.1 \mathrm{~T}, 298 \mathrm{~K}$ and $5 \mathrm{kHz}$ MAS of (f) neat $\alpha-\mathrm{FAPbl}_{3}$ powder and $(\mathrm{g}) \alpha-\mathrm{FAPbl}_{3}$ treated with ADAHI. $\alpha-\mathrm{FAPbl}_{3}$ was prepared mechanochemically and the modulated samples contains $7 \mathrm{~mol} \%$ of ADAHI. Adapted from ref. [50] with permission from Royal Society of Chemistry. (h) Structural representation of a two-dimensional perovskite material with the evolution of the photovoltaic parameters of the corresponding solar cells in ambient air. Adapted with permission from ref. [51] Copyright 2019 American Chemical Society.
[28] X. Li, M. I. Dar, C. Yi, J. Luo, M. Tschumi, S. M. Zakeeruddin, M. K. Nazeeruddin, H. Han, M. Grätzel, Nat. Chem. 2015, 7, 703.

[29] F. Bella, G. Griffini, J. P. Correa-Baena, G. Saracco, M. Grätzel, A. Hagfeldt, S. Turri, C. Gerbaldi, Science 2016, 354, 203.

[30] F. Wang, W. Geng, Y. Zhou, H.-H. Fang, C.-J. Tong, M. A. Loi, L.-M. Liu, N. Zhao, Adv. Mater. 2016, 28, 9986.

[31] X. Zheng, B. Chen, J. Dai, Y. Fang, Y. Bai, Y. Lin, H. Wei, X. C. Zeng, J. Huang, Nat. Energy 2017, 2, 17102.

[32] C. Qin, T. Matsushima, T. Fujihara, C. Adachi, Adv. Mater. 2017, 29, 1603808.

[33] H. Zhang, X. Ren, X. Chen, J. Mao, J. Cheng, Y. Zhao, Y. Liu, J. Milic, W.-J. Yin, M. Grätzel, W. C. H. Choy, Energy Environ. Sci. 2018, 50, 487.

[34] D. Bi, X. Li, J. V. Milić, D. J. Kubicki, N. Pellet, J. Luo, T. LaGrange, P. Mettraux, L. Emsley, S. M. Zakeeruddin, M. Grätzel, Nat. Commun. 2018, 9,4482 .

[35] A. R. Bin Mohd Yusoff, P. Gao, M. K. Nazeeruddin, Coordin. Chem. Rev. 2018, 373, 258 .

[36] J.-P. Correa-Baena, M. Anaya, G. Lozano, W. Tress, K. Domanski, M. Saliba, T. Matsui, T. J. Jacobsson, M. E. Calvo, A. Abate, M. Grätzel, H. Míguez, A. Hagfeldt, Adv. Mater. 2016, 28, 5031.

[37] A. Sharenko, M. F. Toney, J. Am. Chem. Soc. 2016, 138, 463.

[38] J. M. Ball, A. Petrozza, Nat. Energy 2016, 1, 16149.

[39] R. J. Stoddard, F. T. Eickemeyer, J. K. Katahara, H. W. Hillhouse, J. Phys. Chem. Lett. 2017, 8, 3289.

[40] W. Tress, M. Yavari, K. Domanski, P. Yadav, B. Niesen, J.-P. Correa-Baena, A. Hagfeldt, M. Grätzel, Energy Environ. Sci. 2018, 11, 151.
[41] F. C. Hanusch, E. Wiesenmayer, E. Mankel, A. Binek, P. Angloher, C. Fraunhofer, N. Giesbrecht, J. M. Feckl, W. Jaegermann, D. Johrendt, T Bein, P. Docampo, J. Phys. Chem. Lett. 2014, 5, 2791.

[42] M. Saliba, T. Matsui, J.-Y. Seo, K. Domanski, J.-P. Correa-Baena, M. K. Nazeeruddin, S. M. Zakeeruddin, W. Tress, A. Abate, A. Hagfeldt, M. Grätzel, Energy Environ. Sci. 2016, 9, 1989.

[43] C. Yi, J. Luo, S. Meloni, A. Boziki, N. Ashari-Astani, C. Grätzel, S. M. Zakeeruddin, U. Röthlisberger, M. Grätzel, Energy Environ. Sci. 2016, 9, 656

[44] X. Li, D. Bi, C. Yi, J. D. Decoppet, J. Luo, S. M. Zakeeruddin, A. Hagfeldt, M. Grätzel, Science 2016, 353, 58.

[45] W. Huang, J. S. Manser, P. V. Kamat, S. Ptasinska, Chem. Mater. 2016, 28 , 303.

[46] D. J. Kubicki, D. Prochowicz, A. Hofstetter, P. Péchy, S. M. Zakeeruddin, M. Grätzel, L. Emsley, J. Am. Chem. Soc. 2017, 139, 10055.

[47] D. J. Kubicki, D. Prochowicz, A. Hofstetter, S. M. Zakeeruddin, M. Grätzel, L. Emsley, J. Am. Chem. Soc. 2017, 139, 14173.

[48] D. J. Kubicki, D. Prochowicz, A. Hofstetter, M. Saski, P. Yadav, D. Bi, N Pellet, J. Lewiński, S. M. Zakeeruddin, M. Grätzel, L. Emsley, J. Am. Chem. Soc. 2018, 140, 3345 .

[49] D. J. Kubicki, D. Prochowicz, A. Hofstetter, S. M. Zakeeruddin, M. Grätzel, L. Emsley, J. Am. Chem. Soc. 2018, 140, 7232.

[50] M. M. Tavakoli, W. Tress, J. V. Milić, D. Kubicki, L. Emsley, M. Grätzel, Energy Environ. Sci. 2018, 11, 3310.

[51] Y. Li, J. V. Milić, A. Ummadisingu, J.-Y. Seo, J.-H. Im, H.-S. Kim, Y. Liu, M. I. Dar, S. M. Zakeeruddin, P. Wang, A. Hagfeldt, M. Grätzel, Nano Lett. 2019, 19, 150 . 